\title{
Does monitor position influence visual-motor performance during minimally invasive surgery?
}

\author{
Alan D. White, ${ }^{a, b}$ Faisal Mushtaq, ${ }^{a, *}$ Rachael K. Raw, ${ }^{a}$ Oscar T. Giles, ${ }^{a}$ Imogen C. Crook, ${ }^{a}$ James Tomlinson, ${ }^{c}$ \\ Danilo Miskovic, ${ }^{d}$ J. Peter A. Lodge, ${ }^{b}$ Richard M. Wilkie ${ }^{a}$ and Mark Mon-Williams ${ }^{a}$ \\ ${ }^{a}$ School of Psychology, University of Leeds, Leeds, UK; ${ }^{b}$ Department of Hepatobiliary and Transplant Surgery, St James's University \\ Hospital, Leeds, UK; ${ }^{c}$ Health Education Yorkshire and Humber Deanery, University of Leeds, Leeds, UK; ${ }^{d}$ Leeds Institute for \\ Biomedical and Clinical Research, University of Leeds, Leeds, UK
}

${ }^{*}$ Corresponding author at: School of Psychology, University of Leeds, Leeds LS2 9JT, UK. Email: f.mushtaq@leeds.ac.uk

Date accepted for publication: 16 February 2016

\section{Abstract}

Background: In minimally invasive surgery (MIS), the natural relationship between hand and eye is disrupted, i.e. surgeons typically control tools inserted through the patient's abdomen while viewing the workspace on a remote monitor, which can be located in a variety of positions. This separates the location of visual feedback from the area in which a motor action is executed. Previous studies suggest that the visual display should be placed directly ahead of the surgeon (i.e. to preserve visual-motor mapping). However, the extent of the impact of this rotation on surgical performance is unknown. Methods: Eighteen participants completed an aiming task on a tablet PC within a surgical box trainer using a laparoscopic tool in a controlled simulated environment. Visual feedback was presented on a remote monitor located at $0^{\circ}$, $\pm 45^{\circ}$ and $\pm 90^{\circ}$, with order randomised using the Latin Square method. Results: Movements were significantly slower when the monitor was $90^{\circ}$ relative to midline, but spatial accuracy was unaffected by monitor position. Interestingly, the effect of reduced speed in the $90^{\circ}$ condition was transient, decreasing over time, suggesting rapid adaptation to the rotation. Conclusions: We conclude that the angle of the visual display in the context of MIS may require a surgeon to adapt to a changed mapping between visual inputs and motor outputs. While this adaptation occurs relatively quickly, it may interfere with skilled actions (e.g. intracorporeal suturing) in complex surgical procedures.

Keywords: laparoscopy; monitor position; simulation; motor performance; motor learning, sensorimotor adaptation

\section{Introduction}

Minimally invasive surgery (MIS) requires a high level of manual dexterity, often within the context of high-pressured situations (i.e. risk to the patient) and small mistakes can have catastrophic consequences. ${ }^{1}$ Specifically, the processes involved in MIS require the central nervous system (CNS) to produce a congruent mapping between the workspace and hand to execute tasks that require a high level of eye-hand coordination. In contrast to open surgery, where direct observation and manipulation are possible, the natural relationship between hand and eye is disrupted in MIS setups. Surgeons typically control tools that are inserted through the patient's abdomen wall while viewing a camera view of the workspace via a remote display. In this environment, visual information is decoupled from the workspace because the display can be located in a variety of positions and angles relative to the surgeon and studies suggest that altering the monitor location can distort the perception of the operative field. ${ }^{2-6}$ Added complexity arises from camera angle, which may be $0^{\circ}, 30^{\circ}$ or $90^{\circ}$ relative to the laparoscopic tool. As such, head position signals are no longer informative about target location (i.e. the surgeon can be looking in the opposite direction to where the hands are moving), and thus, the surgeon can experience proprioceptive discordance, because the viewing angle of the monitor does not provide useful information about the visual-motor mapping (in contrast to normal visual-motor interactions). ${ }^{7}$

Given the cost of movement errors in surgical environments, understanding how the CNS adapts during MIS tasks is imperative. ${ }^{8}$ Several studies in the surgical literature have suggested that incomplete decoupling of head position signals during surgical tasks results in significant performance costs in MIS. ${ }^{9-13}$ Generally, studies imply that MIS monitors should be positioned in front of the surgeon, and at eye level, in order to minimize inconsistency between the hand and eye. ${ }^{9,13}$ Positioning the monitor head-on to the 
surgeon performing the procedure is also more likely to yield optimal performance ${ }^{12}$ and, as a small-scale study suggests, is also a surgeon's preferred setup. ${ }^{14}$ This setup is not, however, always adopted in operating theatres, and the monitor is often positioned in an oblique manner relative to the surgeon. We were, therefore, interested in how head rotation per se might modulate motor performance. The extant literature has demonstrated (i) performance advantage when the head and hands are pointed in the same direction during visual-motor tasks; (ii) a subjective preference of surgeons for the visual angle to be at $0^{\circ}$; and (iii) an improved ability in experienced surgeons to deal with rotations of the visual display compared with less experienced surgeons. ${ }^{11,12}$ These studies have not quantified the effects of head rotation/viewing angle on visual-motor control processed in MIS and thus the extent to which visual transformations modulate motoric control processes is unclear. Therefore, an empirical investigation into the role of monitor angle on motoric performance in MIS is necessary.

In order to gain a better understanding of how the positioning of visual feedback can modulate motor performance, we used a sensitive Kinematic Assessment Tool (KAT), a validated measure of motor performance that is able to capture a number of performance-related movement variables. ${ }^{15,16}$ We designed an experiment where a tablet PC was placed within a laparoscopic box trainer (LBT; Ethicon, Norderstedt, Germany) and participants used a laparoscopic tool inserted through holes (or ports) in the side of the box to make a series of discrete aiming movements (moving the tip of the tool across the surface of the tablet PC screen). This setup provided a simulation of a laparoscopic setup (i.e. participants could not see what their hands were doing and surgical tools were used to complete the task) as per previous studies, ${ }^{10}$ but provided highly controlled measurements of performance. To establish whether monitor position interfered with performance, speed and accuracy of motor performance were compared when the visual display of targets on a remote monitor was in one of three angular positions $\left(0^{\circ}, \pm 45^{\circ}\right.$ or $\left.\pm 90^{\circ}\right)$. Predicated on past research, ${ }^{2-4,9}$ we hypothesized that increased disparity between monitor angle and torso would result in a systematic decrement in performance. A secondary examination into the effect of monitor position was also carried out through comparison of performance in the first and last trials on the task.

\section{Methods}

\section{Participants}

Eighteen healthy adults took part in this study (10 males). All participants were right-handed as indexed by the
Edinburgh Handedness Inventory (scores $>40$ out of 100 indicate right-hand preference ${ }^{17}$ ). The average age of participants was 24.5 years (range, 21-34 years; SD, 3.7 years). All participants had normal or corrected-to-normal vision, with no history of movement or neurological disorders.

\section{Ethics}

The study was approved by the University of Leeds School of Psychology ethics committee and conducted in accordance with the 1964 Declaration of Helsinki. Participants provided their written full informed consent prior to their involvement in the study and were fully debriefed at the end of the study.

\section{Materials and procedure}

\section{Ethicon laparoscopic box trainer}

The LBT $(390 \mathrm{~mm} \times 265 \mathrm{~mm} \times 180 \mathrm{~mm}$; Fig. 1) was positioned $700 \mathrm{~mm}$ above the floor and rotated $90^{\circ}$ anticlockwise with the shorter sides orthogonal to the supporting table. The LBT had seven entry ports (a diameter of $40 \mathrm{~mm}$ with soft rubber entries in cross hair shapes) positioned in a letter $\mathrm{H}$ configuration. An ENDOPATH XCEL Dilating Tip $12 \mathrm{~mm}$ trocar (Ethicon, Norderstedt, Germany) was fully inserted through each port with the gas valve facing away from the participant. A soft foam section measuring $73 \mathrm{~mm} \times 60 \mathrm{~mm} \times 15 \mathrm{~mm}$ was used as a collar between the port and trocar to allow free range of movement. A Toshiba Portege M700-13P tablet PC (screen $260 \times 163 \mathrm{~mm} ; 1440 \times 900$ pixels; 32 bit colour; $60 \mathrm{~Hz}$ refresh rate) was placed inside the $\mathrm{LBT}$ at the distal right corner, and the built-in touch screen acted as an input device. Participants used a 330-mm-long laparoscopic grasper with plastic tip that was inserted through the trocar and placed on the screen of the tablet PC. The lowest point of the screen was positioned $580 \mathrm{~mm}$ above the table ensuring the display was presented at eye level. The end point of the laparoscopic grasper was represented by an onscreen cursor and controlled by moving across the touch screen and the position was sampled at $120 \mathrm{~Hz}$. Black markers were placed on the floor to indicate where the participants should stand in order to ensure a consistent viewing distance of approximately $800 \mathrm{~mm}$.

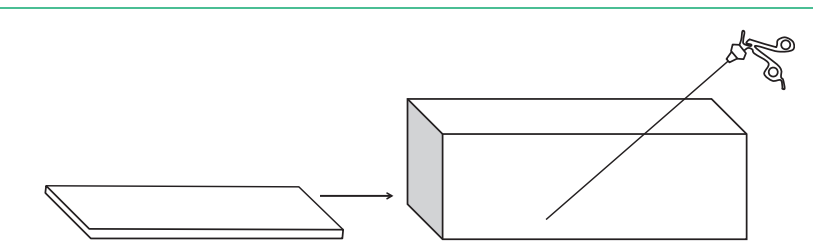

Figure 1 A Toshiba Portege laptop was placed inside the Ethicon laparoscopic box trainer. The laparoscopic tool was used as a stylus (akin to writing on a touch screen) to record kinematic data. 


\section{Visual-motor transformation task}

The experimental task was created using the $\mathrm{KAT}^{16}{ }^{16}$ a validated system for objectively measuring human motor performance in configurable visual-spatial tasks. ${ }^{15,16,18}$ The KAT task in this study was displayed on a tablet PC inside the LBT, and participants were required to make a series of discrete aiming movements between targets that appeared on a remote screen, with a $30^{\circ}$ rotation applied to increase the complexity of the task. Each trial began at the start icon (the green S in Fig. 2), and participants used the laparoscopic grasper to move a cursor from one green dot to the next in a sequential manner. Once a green dot was reached, the next green dot would appear. Participants continued to move from one green dot to the next until they arrived at the red $\mathrm{F}$ for finish icon.

As the stimuli in the aiming task were occluded from direct vision (i.e. the tip of the laparoscopic grasper was occluded inside the LBT), visual stimuli therefore appeared on a Dell $1708 \mathrm{FP}$ monitor (screen $339 \mathrm{~mm} \times 270 \mathrm{~mm}, 1280 \times 1024$ resolution, $75 \mathrm{~Hz}$ refresh rate) positioned at one of three angles (Fig. 3). Participants performed an aiming task trial (i.e. moving from the $\mathrm{S}$ to $\mathrm{F}$ icon) 12 times at each monitor position $\left(0^{\circ}, \pm 45^{\circ}, \pm 90^{\circ} ; 36\right.$ trials in total $)$, and the order in which participants undertook these three conditions was randomised using the Latin Square method.

\section{Analysis}

Three standardized temporal, spatial and frequency metrics were recorded during the aiming task:

(1) Movement time (MT): the time taken to complete a trial of the aiming task trial, as an indicator of movement speed (seconds).
(2) Path length (PL): the length of movement trajectories (millimetres) from start to finish of an aiming task trial, and an indicator of spatial accuracy; longer trajectories indicate disruption to the path of movement, either due to increased motor variability (e.g. shaky hands) or deviation from the straight path between aiming targets.

(3) Normalized jerk (NJ): a marker of the smoothness of movements throughout an aiming task trial; $\mathrm{NJ}$ is the time derivative of acceleration and is minimized in smooth movements. ${ }^{16}$

Mean scores for MT, PL and NJ were calculated across all 12 aiming task trials, and across the first four (F4) and last four (L4) trials, for each monitor position condition $\left(0^{\circ}\right.$ vs. $45^{\circ}$ vs. $\left.90^{\circ}\right)$. Separate repeated measures ANOVAs were applied for each outcome metric recorded on the aiming

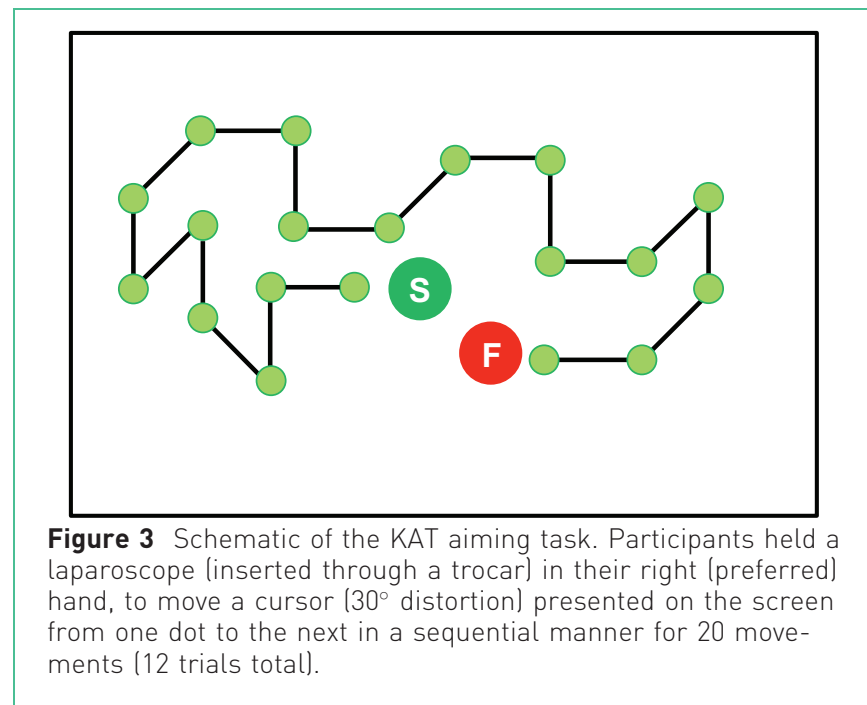

A

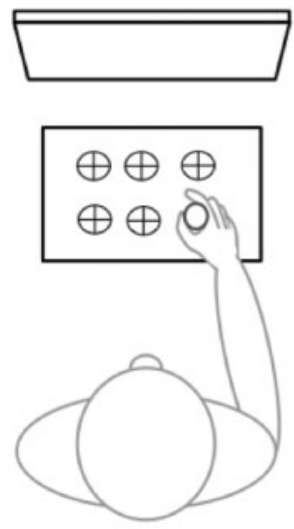

B

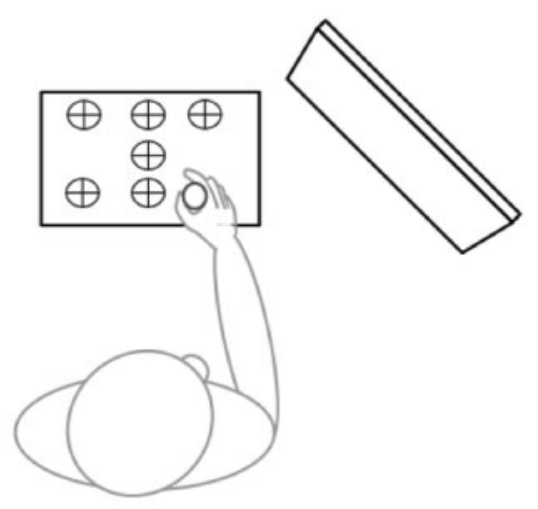

C

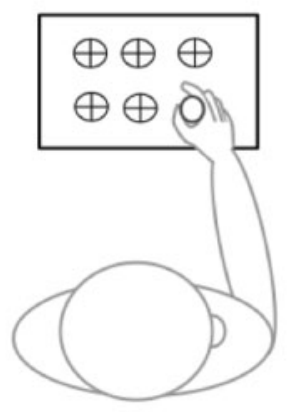

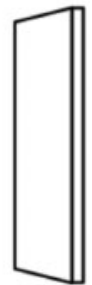

Figure 2 Monitor positions for the experimental aiming task. Examples of the monitor positions for the experimental aiming task whereby the visual feedback was presented in three different positions: (A) $0^{\circ}$ position: visual feedback was presented on a monitor positioned directly ahead of the participant; (B) $45^{\circ}$ position: visual feedback was presented on a monitor positioned at $45^{\circ}$ position relative to body orientation; (C) $90^{\circ}$ position: visual feedback was presented on a monitor positioned at $90^{\circ}$ position relative to body orientation. 
task to examine the effects of monitor position on motor performance $\left(0^{\circ}\right.$ vs. $45^{\circ}$ vs. $\left.90^{\circ}\right)$ and to investigate learning, computed as the difference in performance measures between the beginning (i.e. F4 trials) and end (L4 trials) trial blocks. Power computations used the observed sample size as the basis of the population effect. ${ }^{19}$

\section{Results}

\section{Movement time}

Analysis of MT data revealed the main effects of position $\left(F(2,34)=4.16, P=0.024, \eta_{\mathrm{p}}^{2}=0.2,1-\beta=0.69\right)$ and trial $\left(F(1,17)=22.18, \quad P<0.001, \quad \eta_{\mathrm{p}}^{2}=0.57, \quad 1-\beta=0.99\right)$. Participants were significantly faster at completing the aiming task when the monitor was $0^{\circ}$ from midline (mean path length time $=1.13 \mathrm{~s}, \mathrm{SE}=0.06$ ), compared with the $45^{\circ}$ conditions $($ mean $=1.16 \mathrm{~s}, \mathrm{SE}=0.05)$ and $90^{\circ}$ (mean $=1.25 \mathrm{~s}, \mathrm{SE}=0.06$ ) (see Fig. $4 \mathrm{~A})$. Aiming movements also gained speed towards the end of the task, with significantly faster movements made across the L4 trial block (mean $\mathrm{MT}$ in $\mathrm{F} 4=1.27 \mathrm{~s}, \mathrm{SE}=0.06 ; \mathrm{L} 4=1.13 \mathrm{~s}, \mathrm{SE}=0.05$; Figs $4 \mathrm{~A}$ and $5 \mathrm{~A}$ ). There was a significant position $\times$ trial interaction $\left(F(2,34)=4.18, P=0.024, \eta_{p}^{2}=0.2,1-\beta=0.7\right)$ whereby the effect of position was present in the F4 trials $\left(F(2,34)=5.13, P=0.011, \eta_{p}^{2}=0.23,1-\beta=0.79\right)$ but not in the $\operatorname{L} 4\left(F(2,34)=1.04, P=0.37, \eta_{p}^{2}=0.6,1-\beta=0.06\right)$. Bonferroni post hoc comparisons subsequently showed that this effect of position in the early trials was driven by a significant difference $(P=0.023)$ between the $0^{\circ}$ (mean $=$ 1.15; $\mathrm{SE}=0.06$ ) and $90^{\circ}$ screen positions (mean $=1.40 \mathrm{~s}$; $\mathrm{SE}=0.10)$ and between the $45^{\circ}($ mean $=1.20 \mathrm{~s}, \mathrm{SE}=0.06)$ and $90^{\circ}$ screen positions $(P=0.027)$. There was no significant difference in MT between the $0^{\circ}$ condition and $45^{\circ}$ condition $(P=0.89)$. There were no differences across the L4 trials $(P>0.266)$.

\section{Path length}

PL provides an index of movement accuracy, whereby shorter PLs indicate better spatial accuracy because trajectories are shorter. Observations of the means showed that there was little difference in PL between the monitor position conditions (mean $\mathrm{PL}$ for $0^{\circ}=47.30 \mathrm{~mm}, \mathrm{SE}=0.97$; $45^{\circ}=47.65 \mathrm{~mm}, \quad \mathrm{SE}=0.84 ; \quad 90^{\circ}=49.47 \mathrm{~mm}, \quad \mathrm{SE}=1.42$ ), hence the main effect of position was not significant
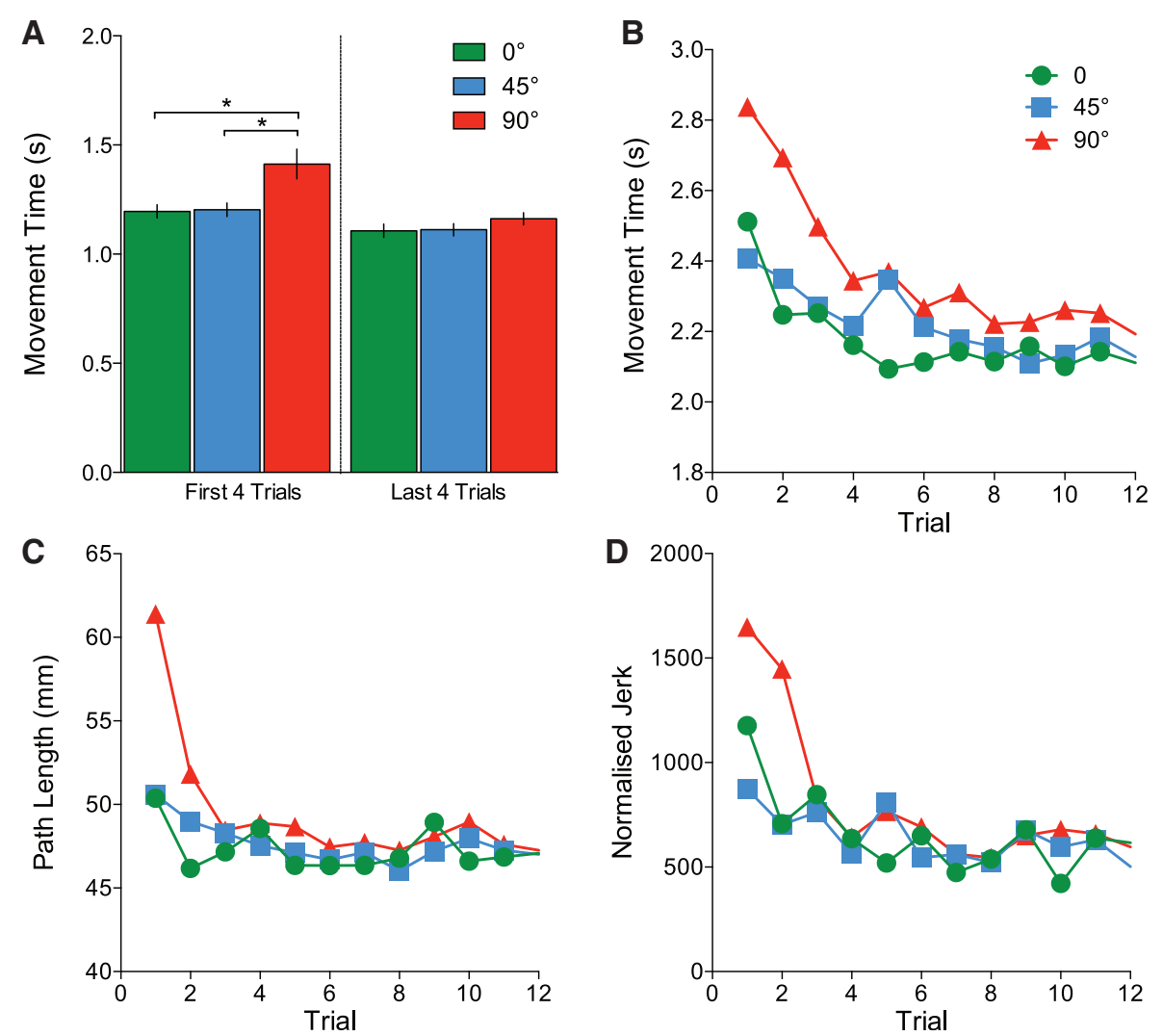

Figure 4 Performance in the aiming task trials. (A) Mean measures of movement time (s) recorded across the first four and last four trials of the aiming task, for each of the three monitor position conditions $\left(0^{\circ}, 45^{\circ}\right.$ and $\left.90^{\circ}\right)$. Error bars represent \pm 1 SEM. Mean measures of motor performance plotted on a trial-by-trial basis for (B) movement time (s); (C) path length (mm); and (D) normalized jerk for each of the three monitor position conditions. 
$(P=0.15)$. Participants did, however, show significant improvements in spatial accuracy as the task progressed; a main effect of trial $\left(F(1,17)=6.2, \quad P=0.02, \quad \eta_{p}^{2}=0.27\right.$, $1-\beta=0.65$; Figs $4 \mathrm{~B}$ and $5 \mathrm{~B})$, revealed shorter PLs in the L4 trials (mean $=47.57 ; \mathrm{SE}=0.091$ ) in comparison with the F4 (mean $=49.0 ; \mathrm{SE}=1.32)$. There was no position $\times$ trial interaction $(P=0.23)$.

\section{Normalized jerk}

Lower NJ values indicate smoother aiming movements. The ANOVA for NJ showed that there was no main effect of position $\left(F(2,34)=1.26, \quad P=0.3, \quad \eta_{p}^{2}=0.07, \quad 1-\beta=0.26\right)$ and no position $\times$ trial interaction $(F(2,34)=2.52, P=0.1$, $\left.\eta_{\mathrm{p}}^{2}=0.13,1-\beta=0.47\right)$. Nevertheless, there was a main effect of trial $\left(F(1,17)=11.85, \quad P=0.003, \quad \eta_{\mathrm{p}}^{2}=0.41\right.$, $1-\beta=0.9$; Figs $4 \mathrm{C}$ and $5 \mathrm{C}$ ), as participants produced increasingly smoother aiming movements towards the end of the task in the L4 trials (mean NJ for $\mathrm{L} 4=611.20$, $\mathrm{SE}=83$ ) compared with the F4 trials (mean PL for $\mathrm{F} 4=903.44, \mathrm{SE}=130.92$ ).

Here we report a comparison of the performance measures between the F4 and L4 trials, but the same pattern of results was obtained when comparing the first and last three trials (see Fig. 4B-D for a trial-by-trial view of the data). To examine more precisely when the initial performance decrement for the $90^{\circ}$ condition had been overcome by participants, we performed a post hoc ANOVA for MT at trial 5 and found that differences were not statistically significant $(P>0.05)$.

\section{Discussion}

One inherent difficulty in MIS is that the viewing angle in these environments does not provide useful information about the visual-motor mapping. The purpose of the present experiment was to quantify the effects of the monitor position on the quality of motor performance in MIS. We also examined the effect of monitor position on motor performance over time. Specifically, we examined the extent to which decrements in performance ameliorate with time (i.e. adaptation through repetitious practice). This was achieved by varying the position of the monitor displaying visual feedback from directly in front of the participant $\left(0^{\circ}\right)$, to a position of $\pm 45^{\circ}$, and $\pm 90^{\circ}$ relative to the body axis. Participants completed a kinematic visual-motor transformation task with a laparoscopic tool, and performance metrics allowed us to assess the extent to which head rotation modulated task performance.

The results showed no difference in motoric performance between the monitor positions of $0^{\circ}$ and $45^{\circ}$. However, consistent with previous studies that have found optimal performance with the monitor at $0^{\circ}$ and significant degradation in performance beyond $45^{\circ},{ }^{3,12,20}$ we observed a significant decrease in performance as indexed by MT in the initial trial in the $90^{\circ}$ condition. PL (an index of accuracy) and NJ (a marker of fluency/smoothness) demonstrated similar trends but did not reach statistical significance thresholds.

Being able to adapt quickly to information presented in different locations and use this input for action has clear advantages for a surgeon who has to simultaneously: (a) deal with complex environments and (b) produce skilled goal-oriented behaviour. In this experiment, we note that the initial decrement in performance when the monitor was positioned at $90^{\circ}$ had almost disappeared by the end of the task. In other words, initial performance was degraded, yet individuals were able to adapt to the new head orientation - a particularly useful process in an MIS environment, when the monitor position can vary from theatre to theatre.

There is a functional relationship between head position and arm movements, ${ }^{21}$ and our data serve to support this assumption. Previous research has demonstrated that neck afferents are important for accurate control of the hand in the absence or degradation of visual-motor information; ${ }^{21,22}$ and vestibular information plays an important role in the control of arm movements. ${ }^{23,24}$ The data we present also add to the literature that has demonstrated the capacity of humans to adapt to visual and mechanical distortions, ${ }^{25,26}$ Adaptation of the human sensorimotor system is, without a doubt, an impressive feat, but the temporary impairment in performance experienced during this process could potentially be problematic in a surgical environment. Specifically, there may be clinical implications for complex MIS procedures where it can be necessary to change the port site through which the camera is passed; or in operations where a re-adjustment of the camera is required. We suggest that it is vital that motor movements are adjusted appropriately in such instances. ${ }^{27}$ Moreover, when a surgeon moves from one operating setup to another (e.g. between an elective theatre to the acute theatre, or from a training simulator to theatre), the monitor orientation should be considered to maintain maximal operative efficiency.

It is worth considering other visual factors that may modulate technical performance in MIS and potentially affect patient safety. The increasing proliferation of virtual environment technologies means that the monitors used in MIS have the potential to supplement natural vision and thus improve performance. For example, displays that present stereoscopic images are becoming increasingly popular in 
MIS and specifically laparoscopy. ${ }^{29,30}$ These systems present surgeons with cues such as depth information, which could be useful for a number of complex surgical tasks as well as more fundamental task such as tying a suture. In robotassisted surgery, where technologies allow for augmenting natural vision, the reverse braille effect has been reported ${ }^{31}$ where sensory integration allows for tactile feedback to be augmented by the enhanced visual information. However, there may be some scenarios in which sustained exposure to displays could have negative consequences. For example, it is known that binocular displays place unnatural pressures on the human oculomotor system ${ }^{32,33}$ and future work should examine the relationship between stereoscopic displays, visual fatigue and discomfort on motor performance in MIS.

\section{Conclusions}

The present work raises an important question regarding surgical training; how can we minimize the negative effects of visuospatial distortions and transformations in MIS? The widespread use of virtual reality simulators, such as the LAP Mentor (Simbionix), ${ }^{26}$ allows students to practice their skills frequently and in a safe environment before entering a reallife scenario. The degree to which simulators provide useful generic training that applies to a variety of surgical tasks is debatable. There are certainly concerns over the extent to which the performance metrics recorded by LAP Mentor reflect a person's real-life level of surgical skill. ${ }^{27}$ Concrete guidelines on how to structure training time (e.g. repeatedly carrying out the same task to gain high-proficiency in an isolated skill versus carrying out a variety of surgical tasks) with simulated systems also do not exist. Critically, a common feature within virtual surgical training schedules is the use of a monitor with a midline-oriented screen (i.e. screen directly in front of the trainee; although distance between monitor and surgeon is much shorter than in theatre). If trainee surgeons practice their skills with this setup, it is highly likely to have a cost when the same task must be carried out when the screen is set off at an angle in theatre. Our results, and those of past studies, lead us to predict that the cost would manifest in reduced motor speed, and this may be amplified in a situation where a surgeon has limited experience. $^{11}$

Structural Learning (SL) theory predicts that learning a surgical technique in a virtual context should transfer to a similar situation in real life if training allows one to learn the fundamental underlying structure of the parameter space, ${ }^{28}$ According to $\mathrm{SL},{ }^{34,35}$ when learning a new skill (e.g. a novel laparoscopic method), the CNS creates a general set of rules that can later be applied and modified when encountering similar scenarios (e.g. a monitor position offset from midline). This process, often described in the cognitive literature as learning to learn (i.e. where common features in a cognitive task are said to facilitate learning of a new but similar task), may be a crucial part of gaining general skills. ${ }^{18}$ In light of our findings, surgeons might be best advised to avoid using monitor positions that deviate from the body midline where possible. In order to ensure that surgical trainees are fully prepared for work in different hospitals/theatres, future research should present trainees with varying monitor display positions in simulation. Based on SL, we predict that this approach will lead to learning that yields adaptability without loss of specificity.

\section{Acknowledgements}

This research project was supported by a grant from the Leeds Teaching Hospitals Charitable Trust.

\section{Conflict of interest}

No conflicts of interest have been declared.

\section{References}

1. White AD, Skelton M, Mushtaq F, Pike TW, MonWilliams M, Lodge JPA, et al. Inconsistent reporting of minimally invasive surgery errors. Ann R Coll Surg Engl 2015; 97: 608-612. doi: 10.1308/rcsann.2015.0038.

2. Holden JG, Flach JM, Donchin Y. Perceptual-motor coordination in an endoscopic surgery simulation. Surg Endosc 1999; 13: 127-132. doi: 10.1007/s004649900920.

3. Matern U, Faist M, Kehl K, Giebmeyer C, Buess G. Montior position in laparoscopic surgery. Surg Endosc 2005; 19: 436-440. doi: 10.1007/s00464-004-9030-7.

4. Conrad J, Shah AH, Divino CM, Schluender S, Gurland B, Shlasko E, et al. The role of mental rotation and memory scanning on the performance of laparoscopic skills: a study on the effect of camera rotational angle. Surg Endosc 2006; 20 : 504-510. doi: 10.1007/s00464-005-0363-7.

5. Moschos E, Coleman RL. Acquiring laparoscopic skill proficiency: does orientation matter. Am J Obstet Gynecol 2004; 191: 1782-1787. doi: 10.1016/j.ajog.2004.07.073.

6. Medina M. Image rotation and reversal: major obstacles in learning intracorporeal suturing and knot-tying. JSLS 1997; 1: 331-336. PMID: 9876698.

7. Lackner JR. Adaptation to visual and proprioceptive rearrangement: origin of the differential effectiveness of active and passive movement. Percept Psychophys 1997; 21: 55-59. doi: 10.3758/BF03199468. 
8. Wann JP, Rushton S, Mon-Williams M. Natural problems for stereoscopic depth perception in virtual environments. Vision Res 1995; 35: 2731-2736. doi: 10.1016/0042-6989(95)00018-U.

9. Hanna GB, Shimi SM, Cuschieri A. Task performance in endoscopic surgery is influenced by location of the image display. Ann Surg 1998; 227: 481-484.

10. Cresswell AB, Macmillan AL, Hanna GB, Cuschieri A. Methods for improving performance under reverse alignment conditions during endoscopic surgery. Surg Endosc 1999; 13: 591-594. doi: 10.1007/s004649901048.

11. Gould JC, Frydman J. Reverse-alignment surgical skills assessment. Surg Endosc 2007; 21: 669-671. doi: 10.1007/s00464006-9135-2.

12. Haveran L, Novitsky Y, Czerniach D. Optimizing laparoscopic task efficiency: the role of camera and monitor positions. Surg Endosc 2007; 21: 980-984. doi: 10.1007/s00464-007-9360-3.

13. Zhang L, Cao CGL. Effect of automatic image realignment on visuomotor coordination in simulated laparoscopic surgery. Appl Ergon 2012; 43: 993-1001. doi: 10.1016/j.apergo.2012. 02.001 .

14. Zehetner J, Kaltenbacher A, Wayand W, Shamiyeh A. Screen height as an ergonomic factor in laparoscopic surgery. Surg Endosc 2006; 20: 139-141. doi: 10.1007/s00464-005-0251-1.

15. Raw RK, Wilkie RM, Culmer PR, Mon-Williams M. Reduced motor asymmetry in older adults when manually tracing paths. Exp Brain Res 2012; 217: 35-41. doi: 10.1007/s00221011-2971-x.

16. Culmer PR, Levesley MC, Mon-Williams M, Williams JHG. A new tool for assessing human movement: the Kinematic Assessment Tool. J Neurosci Methods 2009; 184: 184-192. doi: 10.1016/j.jneumeth.2009.07.025.

17. Oldfield RC. The assessment and analysis of handedness: the Edinburgh inventory. Neuropsychologia 1971; 9: 97-113. doi: 10.1016/0028-3932(71)90067-4.

18. Johnson RL, Culmer PR, Burke MR, Mon-Williams M, Wilkie RM. Exploring structural learning in handwriting. Exp Brain Res 2010; 207: 291-295. doi: 10.1007/s00221010-2438-5.

19. O'Keefe DJ. Brief report: post hoc power, observed power, a priori power, retrospective power, prospective power, achieved power: sorting out appropriate uses of statistical power analyses. Commun Methods Meas 2007; 1: 291-299. doi: 10.1080/ 19312450701641375.

20. Emam TA, Hanna G, Cuschieri A. Comparison of orthodox versus off-optical axis endoscopic manipulations. Surg Endosc 2002; 16: 401-405. doi: 10.1007/s00464-001-8137-3.

21. Berger M, Lechner-Steinleitner S, Kozlovskaya I, Holzmüller G, Mescheriakov S, Sokolov A, et al. The effect of head-to-trunk position on the direction of arm movements before, during, and after space flight. J Vestib Res 1998; 8: 341-354. doi: 10.3233/VES-1998-8501.
22. Guerraz M, Blouin J, Vercher J-L. From head orientation to hand control: evidence of both neck and vestibular involvement in hand drawing. Exp Brain Res 2003; 150: 40-49. doi: 10.1007/s00221-003-1411-y.

23. Bresciani J-P, Blouin J, Popov K, Bourdin C, Sarlegna F, Vercher JL, et al. Galvanic vestibular stimulation in humans produces online arm movement deviations when reaching towards memorized visual targets. Neurosci Lett 2002; 318 : 348. doi: 10.1016/S0304-3940(01)02462-4.

24. Bresciani J-P, Blouin J, Sarlegna F, Bourdin C, Vercher JL, Gauthier GM, et al. On-line versus off-line vestibular-evoked control of goal-directed arm movements. Neuroreport 2002; 13: 1563-1566. PMID: 12218706.

25. Shadmehr R, Brashers-Krug T. Functional stages in the formation of human long-term motor memory. J Neurosci 1997; 17: 409-419. PMID: 8987766.

26. Stratton GM. Vision without inversion of the retinal image. Psychol Rev 1897; 4: 463-481. doi: 10.1037/h0075482.

27. Andreatta PB, Woodrum DT, Gauger PG, Minter RM. LapMentor metrics possess limited construct validity. Simul Healthc 2008; 3: 16-25. doi: 10.1097/SIH.0b013e31816366b9.

28. White AD, Giles O, Sutherland R, Ziff O, Mon-Williams M, Wilkie $\mathrm{R}$, et al. Minimally invasive surgery training using multiple port sites to improve performance. Surg Endosc 2014; 28: 1188-1193. doi: 10.1007/s00464-013-3307-7.

29. Destro F, Cantone N, Lima M. 3D laparoscopic monitors. Med Equip Insights 2014; 5: 9-12. doi: 10.4137/MEI.S13342.

30. Smith R, Day A, Rockall T, Ballard K, Bailey M, Jourdan I. Advanced stereoscopic projection technology significantly improves novice performance of minimally invasive surgical skills. Surg Endosc 2012; 26: 1522-1527. doi: 10.1007/s00464011-2080-8.

31. Tewari AK, Patel ND, Leung RA, Yadav R, Vaughan ED, ElDouaihy $\mathrm{Y}$, et al. Visual cues as a surrogate for tactile feedback during robotic-assisted laparoscopic prostatectomy: posterolateral margin rates in 1340 consecutive patients. BJU Int 2010; 106: 528-536. doi: 10.1111/j.1464-410X.2009.09176.x.

32. Wann JP, Mon-Williams M. What does virtual reality NEED?: human factors issues in the design of three-dimensional computer environments. Int J Human-Computer Studies 1996; 44 : 829-847. doi: 10.1006/ijhc.1996.0035.

33. Mon-Williams M, Wann JP. Binocular virtual reality displays: when problems do and don't occur. Hum Factors 1998; 40: 42-49. doi: 10.1518/001872098779480622.

34. Braun DA, Waldert S, Aertsen A, Wolpert DM, Mehring C. Structure learning in a sensorimotor association task. PLoS One 2010; 5: e8973. doi: 10.1371/journal.pone.0008973.

35. Wolpert DM, Diedrichsen J, Flanagan JR. Principles of sensorimotor learning. Nat Rev Neurosci 2011; 12: 739-751. doi: 10.1038/nrn3112. 